\title{
Anorectal sphincter muscle stimulator design
}

\author{
S. Kara, M. Okandan \\ Dept. of Electronics Engineering. Erciyes University, Turkey.
}

\begin{abstract}
One of the anorectal anomalies observed very commonly in our university hospital is the problem of closed rectum in the newborns. Before operating on the rectal area, a surgeon needs to stimulate the anorectal sphincter muscle in order to prevent any damage to the muscle itself while making a rectal opening. Stimulation of this muscle also helps to locate the rectal opening at the right place; otherwise false positioning of the opening might lead to some other undesirable problems. In this study, we have designed a stimulator that provides reasonably constant output current depending on the physiological impedance of the rectal area. The current amplitude can be set between 20 and $250 \mathrm{mAs}$. Stimulation pulses are adjusted for a duration of $230 \mu$ s and can be obtained at a repetition period between 1.75 and 20 $\mathrm{ms}$. The hardware part is composed of five parts; 1) DC to $\mathrm{AC}$ conversion from battery power supply 2) AC Amplification through a transformer 3) AC to DC conversion 4) Current Regulation and 5) Pulse duration and period adjustment. The stimulator has been tested on 70 patients in the Pediatric Surgery Department of Erciyes University Hospital. On average, about $140 \mathrm{mAs}$ was enough to stimulate the muscle when probes were applied over the skin. On the other hand, with the placement of probes beneath the skin, stimulation was obtained for pulse amplitude of $40 \mathrm{mAs}$. The main advantages of this designed system over the available commercial stimulators are its compact size, light weight and low cost. Best of all, you are not bound with searching for an electrical outlet on the walls, since it is operated on a battery supply and also due to its portability, it can be easily used at multiple sites.
\end{abstract}

\section{Introduction}

One of the anorectal anomalies observed very commonly in our university hospital is the problem of closed rectum in the newborns. In such case, surgical treatment is 
inevitable. Before operating on the rectal area, a surgeon needs to stimulate the anorectal sphincter muscle in order to prevent any damage to the muscle itself while making a rectal opening. Stimulation of this muscle also helps to locate the rectal opening at the right place; otherwise false positioning of the opening might lead to some other undesirable problems such as muscle deformation and wrong localization of anus $[1,2,3]$.

\section{Materials and methods}

In this study, we have designed a stimulator that provides reasonably constant output current depending on the physiological impedance of the rectal area. The current amplitude can be set between 20 and $250 \mathrm{mAs}$. Stimulation pulses are adjusted for a duration of $230 \mu$ s and can be obtained at a repetition period between 1.75 and 20 ms. The hardware part is composed of five parts; 1) DC to $\mathrm{AC}$ conversion from battery power supply 2) AC Amplification through a transformer 3) AC to DC conversion 4)Current Regulation and 5) Pulse duration and period adjustment (Figure 1).

\subsection{DC to AC conversion and AC Amplification through a transformer}

4047 IC, composed of oscillator, astable multivibrator and frequency divider circuits, is supplied by two +9 Volt batteries summing up to 18 Volts DC. This IC provides two square wave output signals, which are inverse of each other and their frequency is adjusted to $50 \mathrm{~Hz}$. These two signals are first passed through speed up capacitors and then fed into to two transistors used for switching purposes. An 18 Volt DC power supply is connected to second pin of primary of the 18 Watts three pin $\mathrm{AC}$ transformer with a multiplying factor of 3 . Collector-emitter junctions of the transistors (Collectors are connected to other two pins of the primary) act as grounding switches as seen in Figure 1. At the secondary of the transformer, 54 Volt, $50 \mathrm{~Hz}$ square wave is obtained [4].

\subsection{AC to DC conversion and Current Regulation}

This square wave is then rectified to a 54 Volt DC via a Wheatstone Bridge rectifier. A capacitor was used to degrade the ripple on the DC. Current regulation is accomplished with the help of a transistor, zener diode and a variable resistor network combination.

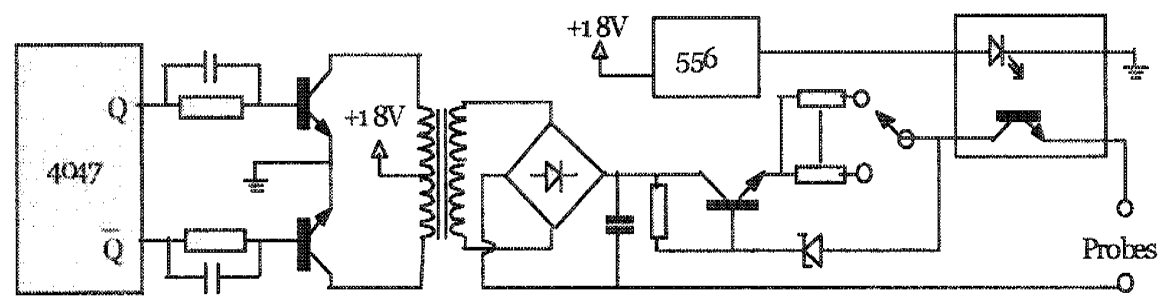

Figure 1: Electronics circuit schematic of the designed stimulator. 
The regulated 54 Volt DC is connected to the collector end of the transistor and a zener diode is used to provide a constant voltage over the base of the transistor to one end of the resistor network, which is connected to emitter of the transistor on the other end. This constant voltage drop over the resistor network supplies an emitter current in steps of $20 \mathrm{mAs}$ from 20 to $250 \mathrm{mAs}$ with the change of the resistance values $[5,6,7]$.

\subsection{Pulse duration and period adjustment}

IC(556) multivibrator is facilitated to drive an opto isolator to turn on/off the current out put. The astable and monostable multivibrators housed in the IC 556 are used to adjust the ON and OFF time of opto isolator, which is composed of light emitting diode and a Phototransistor. In this way, pulse duration and pulse repetition frequency is set. This pulse current output is applied to the patient through two electrode probes, one of which is the reference and the other is the active end $[8,9]$.

The electrodes, in the size of approximately $1.5 \mathrm{~mm}$ thickness, $1.5 \mathrm{~mm}$ wide and $100 \mathrm{~mm}$ length, are open on one end for about $10 \mathrm{~mm}$ to make contact to the skin. The rest is covered with plastic for isolation. The electrode needs to be contacted to skin very firmly to lessen the contact impedance [10].

\section{Results}

The stimulator has been tested on 70 patients in the Pediatric Surgery Department of Erciyes University Hospital. On average, about $140 \mathrm{~mA}$ was enough to stimulate the anorectal sphincter muscle when probes were applied over the skin. On the other hand, with the placement of probes beneath the skin, stimulation was obtained for a pulse amplitude of $40 \mathrm{mAs}$.

\section{Discussion and Conclusion}

The output is limited to a maximum of 54 Volts, because voltage levels higher than that are not needed for stimulation purposes. Parameters of circuit components may be adjusted in order to meet any desired characteristics such as current level, pulse duration and pulse repetition frequency.

The main advantages of this designed system over the available commercial stimulators are its compact size, light weight and low cost. The circuit requires very few components and can be implemented in a considerably short time. The components are cheap and readily available. Especially, sophisticated components are avoided.

Best of all, you are not bound with searching for an electrical outlet on the walls, since it is operated on a battery supply and also due to its portability, it can be easily used at multiple sites. Depending solely on the battery power supply saves the stimulator from being affected by the fluctuations on mains voltage. The stimulator is in routine use in Pediatric Surgery Department and found to be efficient and reliable enough to serve for anorectal sphincter muscle stimulation. 


\section{References}

[1] Freeman, N.V., Burge, D. M., Griffith, B.M. Surgery of Newborn, New York, pp. 111-119, 1994.

[2] Shannon, R., Model of Safe Levels for Electrical Stimulation, $T-B M E$, pp. 424$426,1992$.

[3] Jacobsan, B., Webster, J.G., Medicine and Clinical Engineering, Englewood Cliffs, N.J.: Prentice-Hall, 1977.

[4] Bently, J.P Principles of Measurement Systems, Longman Group, 1983.

[5] Sheingold, D.H., Transducers for Biomedical Measurement Principles and Applications, John Willey \& Sons. Inc., 1974.

[6] Geddes, L.A., Electrodes and the Measurements of Bioelectric Events, New York, :John Willey\& Sons, Inc., 1972.

[7] Gray, P.R., Meyer, R.G., Analysis and Design of Analog Integrated Circuits, John Wiley \& Sons, New-York, 1984.

[8] Floyd, T.L., Electronic Devices, Columbos, OH: Merril Publishing, 1984.

[9] Pearce, J.A., Electro surgery, John Wiley \& Sons, New-York, 1984.

[10] Pfeiffer, J.A., Electrical Stimulation of Sensory Nerves with Skin Electrodes for Research, Diagnosis, Communication and Behavioral Conditioning $A$ Survey, Medical and Biological Engineering, (6), pp. 637-651, 1968. 